\title{
Correction to: Investigation of a Simple Pickup Method for Doping Molecules into Water Clusters
}

\section{Chuanfu Huang ${ }^{1}$}

Published online: 30 July 2018

(C) Springer Science+Business Media, LLC, part of Springer Nature 2018

\section{Correction to: Journal of Cluster Science} https://doi.org/10.1007/s10876-018-1403-5

In the original version of the article, one of the funding information was not provided in acknowledgement section. The correct acknowledgement is given below.
This work was supported by the Fundamental Research Funds for the Central Universities under Grant No. 2018QNB11 and supported by the U.S. National Science Foundation under Grant No. CHE-1213410, and the measurements were taken at the Nanocluster Physics Laboratory at the University of Southern California.

The original article can be found online at https:// doi.org/10.1007/s10876-018-1403-5.

Chuanfu Huang

chuanfuh@cumt.edu.cn

1 College of Physical Science and Technology, China

University of Mining and Technology,

Xuzhou 221116, Jiangsu, China 